\title{
HISTÓRICO DA NEUROLOGIA INFANTIL NA FACULDADE DE MEDICINA DE RIBEIRÃO PRETO -USP
}

\author{
HISTORICAL OF THE PEDIATRIC NEUROLOGY IN THE MEDICAL SCHOOL OF RIBEIRÃO PRETO - USP
}

Maria Valeriana Leme de Moura Ribeiro

Docente. Departamento de Neuropsiquiatria e Psicologia Médica - Faculdade de Medicina de Ribeirão Preto-USP; Departamento de Neurologia da Faculdade de Ciências Médicas - UNICAMP

CoRRESPONDÊNCIA: Caixa Postal 6111. CEP 13083-970 Campinas, SP Tel/ Fax (019) 788-7990. E-mail valerianaribeiro@uol.com.br

MOURA RIBEIRO MVL. Histórico da neurologia infantil na Faculdade de Medicina de Ribeirão Preto-USP. Medicina, Ribeirão Preto, 33: 88-92, jan./mar. 2000.

RESUMO: São apresentados os principais dados históricos relacionados à criação e organização da Neurologia Infantil no Departamento de Neuropsiquiatria e Psicologia Médica da Faculdade de Medicina de Ribeirão Preto, USP, no período de 1960 - 1990. São analisadas as interações com os vários departamentos e professores co-paticipantes no trabalho assistencial, de pesquisa e ensino, com enfase aos aspectos relacionados à implantação e desenvolvimento da Residência Médica em Neurologia Infantil

UNITERMOS: Neurologia. Pediatria. Internato e Residência; hstória.

A partir de novembro de 1962, sob o incentivo do Professor Jorge Armbrust de Lima Figueiredo, foi criado e organizado o setor de Neurologia Infantil do Hospital das Clínicas, junto ao Departamento de Neurologia da Faculdade de Medicina de Ribeirão Preto da Universidade de São Paulo (FMRP-USP ).

Naquela época, o Departamento de Neurologia da Faculdade de Medicina da USP, em São Paulo, com objetivos educacionais bem definidos, já desenvolvia programação sistematizada de assistência, ensino e pesquisa em Neurologia Infantil, sob a liderança do Professor Antônio Branco Lefèvre, que procurava mostrar a qualidade e a diferenciação dos médicos internos que atuavam na área.

O Professor Jorge Armbrust Figueiredo, com formação complementar em Neurologia, nos Estados Unidos, e o Professor J. Renato Woiski, visitante regular dos Centros Universitários em diversos países da Europa, valorizaram a então emergente Neurologia Infantil como especialidade integrante das Ciências
Neurológicas, por entenderem que os conhecimentos sobre o sistema nervoso da criança em desenvolvimento deveriam ser mediados pelo raciocínio do especialista em Neurologia, alicerçando, legítima e consistentemente, os conhecimentos sobre o cérebro infantil. Portanto, a Neurologia Infantil, na Faculdade de Medicina de Ribeirão Preto, a partir da atuação de professores eminentemente clínicos, interessados na evolução das pesquisas, teve a oportunidade de desenvolver e dinamizar não somente estudos relacionados às diferentes anormalidades neurológicas, como, também, a respeito de metabolismo e nutrição na criança, sendo avaliada sua repercussão sobre o desenvolvimento neuropsicomotor e cognitivo $^{(1 / 4)}$.

No antigo Hospital das Clínicas, hoje denominado Unidade de Emergência, foi iniciada, em 1961, pelo Departamento de Neurologia da FMRP - USP, a programação de Residência, segundo as normas da instituição, sendo a primeira residente $\left(1^{\circ}\right.$ e $2^{\circ}$ anos), Maria Valeriana Leme de Moura Ribeiro. Nessa ativi- 
dade, os docentes do Departamento de Neurologia prestavam assessoria ao ensino e atendimento de crianças com distúrbios neurológicos, enfatizando a importância da semiologia específica da criança em desenvolvimento, procurando caracterizar o diagnóstico sindrômico, topográfico e etiológico, envolvendo, sempre, conhecimentos de neuroanatomia, neurofisiologia e neuropatologia entre outros.

No período de 1960 - 1964, as crianças com anormalidades neurológicas eram internadas nas Enfermarias de Pediatria, e, a partir de 1964, na Enfermaria de Neurologia Infantil, com seis leitos, anexa à enfermaria de Neurologia Geral de adultos, com 22 leitos, mantendo ativo, também, o atendimento das interconsultas nas enfermarias das diversas especialidades.

Em junho de 1963, o Departamento de Neurologia da FMRP - USP, contratou-nos como instrutora, em Regime de Dedicação Integral à Docência e Pesquisa (RDIDP), com o objetivo, já concretizado, de assistência, ensino, pesquisa e administração da Neurologia Infantil.

Em colaboração com a Dra. Terezinha Cresci Braga Montelli, contratada em 1967 pelo mesmo departamento, foi organizado o setor de avaliação do desenvolvimento neuropsicomotor e cognitivo aplicado à Neurologia, visando ao atendimento de crianças com distúrbios do comportamento e do aprendizado. Nessa ocasião, novas áreas do conhecimento, irradiadas de países desenvolvidos, passaram a influenciar os anseios científicos de grandes mestres brasileiros, que providenciaram a implantação dos cursos de PósGraduação, mediante esforço conjunto de departamentos e disciplinas. Assim, na FMRP-USP, foi organizado o primeiro curso de Pós-Graduação sobre "Metabolismo e Nutrição", envolvendo os Departamentos de Bioquímica, Genética, Fisiologia, Pediatria e Estatística, com início em 1966 e término em 1968, condicionando uma inovação segura na formação de docentes pesquisadores nos diferentes campos de estudos. As primeiras pesquisas, envolvendo professores de vários departamentos, foram realizadas, versando sobre desenvolvimento neuropsicomotor em crianças desnutridas, estudo evolutivo de crianças que tiveram meningencefalites bacterianas, e estudos laboratoriais em genética médica.

Em 1969, ao Departamento de Neurologia, integraram-se a Psiquiatria e a Psicologia Médica (Departamento de Neuropsiquiatria e Psicologia Médica NPPM), iniciando a fase de estudos e reuniões científicas, multidisciplinares de Neuropsiquiatria Infantil, envolvendo neurologistas, psiquiatras, psicólogos, psicopedagogos, contando com a colaboração ativa da Prof ${ }^{a}$. Hona Tahim Mantovani, da Prof ${ }^{a}$. Edna Maria Maturano e do Prof. José Hércules Golfeto.

Um fato merecedor de registro foi a participação da Neurologia Infantil de Ribeirão Preto nas atividades da Associação Brasileira de Neurologia e Psiquiatria Infantil, ABENEPI, fundada em 1967. Já, naquela época, a $\mathrm{Dr}^{\mathrm{a}}$. Valeriana foi responsável pela dinamização científica da Associação na região de Ribeirão Preto, numa demonstração de apoio da Diretoria da ABENEPI à Neurologia Infantil da FMRP - USP ${ }^{(5)}$.

Fazendo parte de um centro universitário no interior do Estado de São Paulo, o setor de Neurologia Infantil buscava orientações para os pacientes através de discussões conjuntas com a cooparticipação científica dos docentes dos vários departamentos (Neurologia, Pediatria, Genética Médica e Moléstias Infecciosas). As reuniões bibliográficas, o estágio regular junto ao Laboratório do Serviço de Líquido Cefalorraquiano - LCR (Prof. Edymar Jardim), junto à Genética Médica (Prof ${ }^{a}$. Iris Ferrari), além de reuniões neuropatológicas (Prof. Richardo Antonio Gallina) e neuropediátricas (várias publicadas na Revista Pediatria Prática), favoreciam o aprendizado, beneficiado pelo crescente interesse nos estudos da eletrencefalografia clínica (EEG) em crianças. Nessa atividade, houve a participação de destaque dos Professores Mário L. Martinez, Rubens Moura Ribeiro, Terezinha de Cresci Braga Montelli e Michel P. Lison, que atuaram com pionerismo, valorizando a análise da organização da atividade elétrica cerebral em crianças, particularizando os padrões eletrofisiológicos relacionados às várias idades, a partir do recém-nascido a termo e pré termo. Em um momento especial, coincidindo com o surto de meningencefalites, poliomielite e sarampo, foram implantados vários protocolos e ambulatórios especiais de pesquisas com avaliação clínica evolutiva, liquórica e de EEG. O acompanhamento, a longo prazo, dos pacientes forneceu subsídios para a elaboração de inúmeros trabalhos apresentados em congressos, além de teses e publicações em periódicos especializados, nacionais e internacionais, permitindo, assim, a divulgação de conhecimentos junto aos alunos de graduação, residentes e pós-graduandos, por uma equipe de trabalho, dinâmica e coesa. Portanto, o esforço conjunto dos professores proporcionou o progresso da Neurologia Infantil, não somente da Faculdade de Medicina de Ribeirão Preto, USP, como, também, da Faculdade de Medicina de Botucatu, UNESP, 
com a transferência da Dra ${ }^{\mathrm{a}}$. Terezinha de Cresci Braga Montelli para o Departamento de Neurologia da referida instituição, em 1968.

Para responder às necessidades da instituição e ampliar os conhecimentos dirigidos à Neurologia Infantil, a Professora M. Valeriana Leme de Moura Ribeiro, em várias oportunidades, visitou e participou de atividades clínicas no Serviço do Prof. Antônio Branco Lefèvre; USP- São Paulo; visitou, também, em 1966, o Serviço de Neurologia Infantil do Instituto de Neurologia do Hospital de Clínicas de Montevidéu (Uruguai), dirigido pela Prof ${ }^{a}$. Maria Antonieta Rebollo; em 1970, o setor de Neurologia Infantil do Hospital For Sick Children, em Londres (Inglaterra), e o Laboratório de Pesquisa em Moléstias Metabólicas, dirigido pela Prof ${ }^{a}$. Rosemary Stephens; em 1970 e em 1973, visitou o Serviço de Neuropediatria do Hospital du Nord, em Marseille (França), sob orientação de Mlle, Pinsard e o Serviço de Eletrencefalografia do Prof. Henri Gastaut no Centre Hospitalaire Universitaire (Hopital de La Timone) em Marseille (França). Em 1975, participou do I International Congress of Child Neurology, em Toronto, Canadá. Com o objetivo de desenvolver os conhecimentos sobre o sistema nervoso em desenvolvimento, seja em seus aspectos anatômicos, funcionais e semiológicos, bem como os mecanismos fisiopatológicos nas diferentes anormalidades, organizou o programa teórico para médicos residentes de Neurologia até o ano de 1974 e, a seguir, para médicos residentes de Neurologia Infantil, a partir de 1975.

No ano de 1975, foi organizado e implantado o Programa de Residência Médica em Neurologia Infantil, (uma vaga), no Departamento de Neuropsiquiatria e Psicologia Médica do Hospital das Clínicas da FMRP - USP, com duração de dois anos. Obedecendo, porém, às diretrizes estabelecidas pela Comissão Nacional de Residência (CNRM), normatizada, no Brasil, através do Decreto-lei no 80281 de 05 de julho de 1977, foi reformulado o conteúdo programático, obedecendo às novas exigências da CNRM. Portanto, a atividade assistencial, o ensino e a pesquisa, até então, realizados pela única docente, $\mathrm{Dr}^{\mathrm{a}}$. Maria Valeriana Leme de Moura Ribeiro, passaram a ser desenvolvidos, também, como parte do aprendizado, pela primeira residente oficial de Neurologia Infantil, Dra . Áurea Nogueira de Melo. Essa nova etapa proporcionou o reconhecimento da Neurologia Infantil como especialidade, com ampliação da atividade assistencial aos recémnascidos do Berçário, e, como decorrência, um incre- mento das pesquisas em Neurologia do Desenvolvimento; permitiu, ainda, a dinamização das atividades assistenciais nos ambulatórios gerais e especializados, condicionando contínuo enriquecimento científico, resultando, disso, inúmeras publicações em periódicos nacionais e internacionais.

Em 1978, inaugurado o novo Hospital das Clínicas, no "Campus" de Monte Alegre, outras atividades foram implantadas e desenvolvidas, sendo mantido, porém, o atendimento a crianças com anormalidades neurológicas na, Unidade de Emergência (UE-cidade) e nas Disciplinas de Psicologia Médica e Psiquiatria (antigo Hospital Emboaba).

Em 1981, houve remodelações dos Programas de Formação em Residência Médica Nacional (Lei 6932), com introdução, na Neurologia Infantil, do prérequisito em Pediatria e, nesse novo sistema, outros médicos foram, anualmente, selecionados para o treinamento em Residência de Neurologia Infantil, obedecendo a diretrizes com progressivo amadurecimento do julgamento clínico, sempre com supervisão docente. A formação competente de inúmeros médicos resultou no credenciamento do Serviço de Neurologia Infantil, pela Comissão Nacional de Residência Médica, em 1982, beneficiando os profissionais provenientes de vários estados: Rio Grande do Norte, Áurea Nogueira de Melo, 1974; Minas Gerais, Maria Helena Zago Lorenzato, 1978; Pará, José Luiz Salame Guimarães, 1979; Bahia, Alfredo Leboreiro Fernandes, 1980; São Paulo, Carolina Araújo Rodrigues Funayama, 1980; Minas Gerais, Katia Maria Martins, 1981; Sergipe, André Almeida, 1981; São Paulo, Antônio Naburo Saito, 1982;São Paulo, Marlene Fátima Turcato, 1982.

Em função do levantamento de dados, evidenciando volume expressivo de atendimento de crianças com doenças neurológicas, do aumento das atividades de docência e de pesquisas, justificamos ao Conselho do Departamento de Neuropsiquiatria e Psicologia Médica e ao setor Administrativo do Hospital das Clínicas da FMRP a necessidade da admissão de médicos com título de especialista em Neurologia Infantil, sendo a Dra . Áurea Nogueira de Melo contratada em fevereiro de 1976 e a $\mathrm{Dr}^{\mathrm{a}}$. Carolina Rodrigues Funayama em outubro de 1981, médicas de grande capacidade profissional e excepcionais predicados morais.

A partir de 1980, a Professora Carolina Rodrigues Funayama e a Professora M. Valeriana Leme de Moura Ribeiro, estudando os erros inatos do metabolismo, passaram a contribuir na confirmação do 
diagnóstico através de exames especializados, em várias doenças metabólicas (Hiperfenilalaninemia, Hiperglicinemia e Leucinóse), tarefa essa auxiliada pela implantação do Laboratório de Química das Proteínas, sob orientação do Professor Lewis Greene.

Foram, também, desenvolvidas pesquisas em colaboração com a Professora Carolina Funayama, em recém- nascidos do Berçário, e com o Professor Alfredo Leboreiro Fernandez (Professor de Neurologia Infantil na Faculdade de Medicina do Triângulo Mineiro), em crianças com neuroinfecções.

Em março de 1980, juntamente com o Dr. Antonio Fernando Reginato, coordenamos a Jornada de Neurologia e Neurocirurgia, patrocinada pela Associação Paulista de Medicina, pela ABENEPI e pelo Clube de Ciências Neurológicas do Interior, realizada na cidade de Barra Bonita, com participação de numerosos especialistas.

O crescimento geral do setor, a produtividade científica e a ampliação do espaço profissional foram conseguidos, contornando-se, com habilidade e agilidade, as dificuldades mediante esforços e dedicação de um grupo de profissionais que acreditaram na importância da especialidade.

Em 1981, foi possível a organização da Primeira Reunião Científica de Neurologia Infantil, em Ribeirão Preto, patrocinada pelo Departamento de Neuropsiquiatria e Psicologia Médica da FMRP-USP, e pelo Centro de Estudos e Pesquisas Pediátricas (Presidente Dr. Salim Moysés Jorge) sob a coordenação das Dras. Maria do Rosário Leme Brasil e M. Valeriana Leme de Moura Ribeiro. Nesse evento, o Prof. Antônio B. Lefèvre e seus colaboradores da USP - São Paulo, em conjunto com Professores da FMRP, proporcionaram atualização dos temas de interesse para expressivo número de médicos da região de Ribeirão Preto.

Novos eventos foram organizados pelo setor de Neurologia Infantil da FMRP - USP, com patrocínio da Associação Brasileira de Neurologia e Psiquiatria Infantil (ABENEPI): 1- "Jornada sobre Cefaléia na Infância", em julho de 1987; 2- curso sobre "Depressão na Infância", em agosto de 1987; 3- curso de "Sono na Criança", em maio de 1988.

A partir de 1990, a Neurologia Infantil da FMRP contribuiu com a organização de eventos regionais ligados às APAES; a partir de 1993, com a de Congressos Anuais do Capítulo Paulista da ABENEPI e, a de eventos da Sociedade Brasileira de Neurologia Infantil (SBNI) e da Academia Brasileira de Neurologia.
Em 1989, como relatora do seminário: "Requisitos Mínimos de um Programa de Residência Médica: Competências em Neurologia Infantil”, a Professora M. Valeriana e a relatora auxiliar, Professora Carolina R. Funayama, concluíram importante trabalho junto ao CNRM e FUNDAP, sendo publicado documento das diretrizes sobre Residência Médica em Neurologia Infantil, com formação definida em quatro anos ${ }^{(6)}$. Devemos ressaltar que o Programa de Residência Médica em Neurologia Infantil sempre foi particularmente privilegiado no HCRP, em função do especial interesse de todos os docentes do Departamento em se dedicar à pesquisa e à assistência de crianças com anormalidades neurológicas.

Em 1988, a Professora M. Valeriana aceitou o convite para orientar a Disciplina de Neurologia Infantil do Departamento de Neurologia da Faculdade de Ciências Médicas da UNICAMP, contando com a especial colaboração das Professoras Ana Maria Sedrez G. Piovesana, Marilisa Mantovani Guerreiro, Sylvia Maria Ciasca e Vanda Maria Gimenes Gonçalves, docentes contratadas em RDIDP.

Assim, na FMRP-USP, com uma equipe pequena, trabalhando em regime de dedicação exclusiva, foi possível elaborar, no período de 1960 a 1990, numerosos trabalhos de pesquisas, com apresentação em Congressos Médicos e publicações em revistas indexadas, de circulação nacional (47 artigos) e internacional (4 artigos), além de colaborar na formação de 23 médicos especialistas em Neurologia Infantil.

A partir de 1990, o Serviço de Neurologia Infantil ficou sob a responsabilidade da Prof ${ }^{a}$. Carolina Araujo Rodrigues Funayama, que, ali, vem mantendo o incentivo à interdisciplinariedade, visando ensino $\mathrm{e}$ pesquisa em neurodesenvolvimento e em suas patologias, orientando na assistência adequada à criança com anormalidades neurológicas. Com esses objetivos, vêm se fortalecendo, aos poucos, as equipes para ensino, pesquisa e assistência nas áreas de neuroneonatologia, neurodesenvolvimento, distúrbios do comportamento, aprendizagem (Prof ${ }^{\mathrm{a}}$. Carolina AR. Funayama) e erros inatos do metabolismo (Dra. Marlene de Fátima Turcato). $\mathrm{O}$ atendimento, na Unidade de Emergência, especialmente valorizado do ponto de vista assistencial e ensino, mantém-se, até hoje, por Médicos do Programa de Residência em Neurologia Geral e/ou da Neurologia Infantil. Somente no ano de 1999, foi contratada a primeira médica, Dr ${ }^{\mathrm{a}}$. Carla Caldas, com formação neuropediátrica, para atuação naquela importante unidade. 
Por fim, devemos ressaltar que o Programa de Residência Médica em Neurologia Infantil é particularmente privilegiado no HCRP, uma vez que conta com a peculiaridade do interesse de todos os docentes do departamento em se dedicar à pesquisa e assistência em áreas neuropediátricas. Atualmente, há ambulatórios especializados somente para atendimento, de crianças com epilepsia. O setor de eletrofisiologia dispõe, hoje, de polissonografia- EEG neonatal, implantado pela atual chefe do laboratório, Prof ${ }^{a}$. Regina Maria França Fernandes. Ressaltamos, ainda, o grande empenho do Dr. Vitor Tumas, especialista em distúrbios do movimento, que há três anos orienta ambulatório destinado apenas às crianças com esse comprometimento. Também os setores de Doenças Neuromusculares (Prof. Amilton Antunes Barreira) e de Cefaléias (Prof. José Geraldo Speciali), bem como o Centro de Cirurgia de Epilepsias (Prof. Américo C. Sakamoto) e, mais recentemente, o de Neurogenética (Prof. Wilson Marques
Júnior), com equipe constituída por vários docentes e médicos assistentes, desenvolvem pesquisas e assistência diferenciada em patologias infantis ${ }^{(4)}$.

Difícil pensar em crescimento, produtividade e melhorias sem conhecer as reais dificuldades e os caminhos percorridos por todos os dedicados profissionais que nos antecederam. Devemos, portanto, estimular os recém-chegados na especialidade para a realização da sua própria história, base fundamental para a compreensão dos fatos presentes e motivo para a perpetuação da luta para reconhecimento, respeito e valorização da Neurologia Infantil.

\section{AGRADECIMENTOS}

À Professora Terezinha Cresci Braga Montelli, Professora Carolina Rodrigues Funayama, Professor Alfredo Leboreiro Fernandez e Professora Áurea Nogueira de Melo pelas sugestões para elaboração deste trabalho.

MOURA RIBEIRO MVL. Historical of the Pediatric Neurology in the Medical School of Ribeirão Preto-USP. Medicina, Ribeirão Preto, 33: 88-92, jan./march 2000.

ABSTRACT: The author present the main historical data related to the creation and organization of the Pediatric Neurology in the Neuropsychiatric and Medical Psychology of the Medical School of Ribeirão Preto, USP, in the period of 1960 - 1990. It has been evaluated the interactions between the several departments and teachers sharing the assistencial work, research and teaching, with emphasis regard to the implantion and development of the Medical Residence in Pediatric Neurology.

UNITERMS: Pediatrics. Neurology. Intership and Residency; history.

\section{REFERÊNCIAS BIBLIOGRÁFICAS}

1 - MOURA-RIBEIRO MVL. Memorial. Concurso de Livre Docência em Neurologia, - Faculdade de Medicina de Ribeirão Preto da USP, Ribeirão Preto, 1978.

2 - FACULDADE DE MEDICINA DE RIBEIRÃO PRETO DA USP. DEPARTAMENTO DE NEUROPSIQUIATRIA E PSICOLOGIA MÉDICA. Medicina, Ribeirão Preto, 25: 113-120, 1992.

3 - LISON MP. Ribeirão Preto. In: REIMÃO R \& ALONSO-NIETO $\mathrm{JL}$, eds. História da neurologia no Estado de São Paulo, Lemos Editorial, São Paulo, p. 139-147, 1996.

4 - MOURA-RIBEIRO MVL. Memorial de Concurso em Neurologia - Categoria PE - III - Docente. Faculdade de Ciências Médicas da Unicamp, Campinas, 1999.
5 - ASSUMPÇÃO Jr FB. Psiquiatria infantil brasileira: um esboço histórico, Lemos Editorial, São Paulo, p. 85, 1995.

6 - MOURA-RIBEIRO MVL \& FUNAYAMA CAR. Requisitos Mínimos de um Programa de Residência Médica: Competência em Neurologia Infantil. Documento de Trabalho FUNDAP, DT 47, São Paulo, 1991.

Recebido para publicação em 10/01/2000

Aprovado para publicação em 03/03/2000 